Journal of Computer Science 5 (2): 115-122, 2009

ISSN 1549-3636

(C) 2009 Science Publications

\title{
Evaluation of the Severity of Mitral Valvular Regurgitation with Doppler Echocardiography Using Proximal Flow Convergence Method
}

\author{
${ }^{1}$ Pinjari Abdul Khayum, ${ }^{2}$ M.N. Giriprasad and ${ }^{3}$ P.V. Sridevi \\ ${ }^{1}$ Department of Electronics and Communication Engineering, \\ Madina Engineering College, Kadapa, Andhra Pradesh, India \\ ${ }^{2}$ Department of Electronics and Communication Engineering, \\ Jawaharlal Nehru Technological University, College of Engineering, \\ Pulivendula, Kadapa District Andhra Pradesh, India \\ ${ }^{3}$ Department of Electronics and Communication Engineering, \\ Andhra University, Andhra Pradesh, India
}

\begin{abstract}
Problem statement: Valvular regurgitation is recognized as the central cause of morbidity and mortality. Even though the clinician can detect the presence of regurgitation by mere physical examination; diagnostic methods become inevitable while estimating the severity of valvular regurgitation and in the transformation of cardiac chambers as in reaction to the volume overload condition. Lately, a promising new technology, the Echocardiography with Doppler is found to facilitate the non-invasive recognition and assessment of the severity and etiology of valvular regurgitation. Accurate measurements of regurgitant volume in patients is of utmost importance since it aids in the estimation of the progression of the disease which in turn is vital for determining the optimal time for surgical repair or replacement. Approach: Color space conversion and anisotropic diffusion segmentation techniques are utilized in this study for the pre-processing stage of the quantification of mitral regurgitation. Flow field measurements are carried out with the aid of proximal flow convergence method. Results: A calculated value of flow rate, regurgitant orifice area, regurgitant fraction and the regurgitant volume for a regurgitant orifice in the cardiovascular system are obtained from the potential Color Doppler visualization of the flow convergence region. Conclusion: The research proposed provides a significant assessment of the echocardiographic and Doppler techniques employed in the evaluation of mitral valvular regurgitation in the patients. Additionally it also proffers the estimation of mildness, severity and eccentricity of mitral valvular regurgitation on basis of the scientific literature and a consensus of a panel of experts.
\end{abstract}

Key words: Echocardiogram, color doppler, mitral regurgitation, anisotropic diffusion, flow convergence, orifice area, flow rate, regurgitant volume

\section{INTRODUCTION}

Medical imaging is referred to as the measures and procedures that are utilized to generate images of the human body (or parts thereof) for clinical purposes (medical procedures seeking to reveal, diagnose or examine disease) or medical science. Broadly medical imaging is a division of biological imaging that encompasses radiology (in the wider sense), radiological sciences, endoscopy, medical thermography, medical photography and microscopy (e.g., for human pathological investigations) ${ }^{[1]}$. Measurement and recording techniques that do not generate image results principally, like the electroencephalography (EEG) and magneto encephalography (MEG) and others, but generate data that can be represented as maps can be classified as kinds of medical imaging ${ }^{[2]}$. Contemporary imaging technologies include Computed Tomography, Positron Emission Tomography (PET), Magnetic Resonance Imaging (MRI) ${ }^{[3]}$ and Ultrasound (Echocardiogram).

The visualization of muscles and internal organs, their size, structures and possible pathologies or lesions of the heart assisting in the detection of a considerable number of heart problems can be achieved with the aid of echocardiogram. Echocardiography aids in the

Corresponding Author: Pinjari Abdul Khayum, Department of Electronics and Communication Engineering, Madina Engineering College, Kadapa, Andhra Pradesh, India 
diagnosis of cardiovascular system besides making precise assessment of the velocity of blood and cardiac tissue at any given point using wither pulsed or continuous wave Doppler ultrasound. This permits the estimation of cardiac valve areas and function, abnormal communications between the left and right side of the heart, leaking of blood through the valves (valvular regurgitation) $^{[8]}$ and determination of the cardiac output in addition to the ejection fraction. Flowrelated measurements can be enhanced by combining it with Doppler ultrasound. At present, Doppler echocardiography plays a vital role in the premature determination of the kind of surgery to be carried out for adjusting the mitral valve regurgitation ${ }^{[5,7]}$.

The severity of the regurgitation is the vital determinant of the development of ventricular dilatation and dysfunction in case of mitral regurgitation [a valvular heart disease also known as mitral insufficiency]. Thus the precise estimation of regurgitant volume in patients having mitral regurgitation is vital for determining the progression of the disease that in turn is beneficial in determining the optimal time for surgical repair or replacement ${ }^{[9]}$. Diverse analytic approaches and diagnostic technologies have been proposed to help in the clinical assessment of mitral regurgitation. Nevertheless, all techniques in existence have displayed limitations in one form or another.

Our contribution: The aim of this research is to present a new technique based on an Image processing method which can accurately quantify the percentage of backward flow of blood, regurgitant flow rate, regurgitant volume, effective regurgitant orifice area, regurgitant fraction and orifice area in mitral regurgitation by using the Doppler Echocardiography image that works on the color flow Doppler mapping methods like proximal flow convergence. In the preprocessing stage, the color Doppler echocardiography image with $\mathrm{RGB}$ color space has been converted into $\mathrm{YC}_{\mathrm{b}} \mathrm{C}_{\mathrm{r} . \text {. }}$ Subsequently it has been segmented with the aid of non linear anisotropic diffusion method that is used to calculate the percentage of backward flow of blood. The proximal flow convergence method has been exploited to quantify valvular regurgitation by the analysis of the converging flow field proximal to assess the mildness, severity and eccentricity of a mitral regurgitant lesion. Furthermore this research offers a review of Qualitative and quantitative parameters useful in grading the mitral regurgitation severity and utility, advantages and limitations of Echocardiographic and Doppler parameters used in the evaluation of mitral regurgitation severity. Besides that a brief introduction about the concepts: Regurgitation, Mitral Regurgitation, Doppler Echocardiography, Color Doppler and anisotropic diffusion segmentation are detailed respectively.

Regurgitation: Regurgitation is the backward flowing of Blood (flow of blood in the cardiovascular system $)^{[11]}$. Mathematically, blood flow is described by Darcy's law (which can be viewed as the fluid equivalent of Ohm's law) and approximately by HagenPoiseuille equation:

Darcy's Law $F=\Delta \mathrm{P} / \mathrm{R}$

Hagen-Poiseuille Equation $R=\left(v L / r^{4}\right)(8 / \pi)$

Where:

$\mathrm{F}=$ Blood flow

$\mathrm{P}=$ Pressure

$\mathrm{R}=$ Resistance

$v=$ Fluid viscosity

$\mathrm{L}=$ Length of tube

$\mathrm{r}=$ Radius of tube

Regurgitation can be categorized into five. They are Aortic ${ }^{[12]}$, Mitral, Pulmonic ${ }^{[10]}$, Tricuspid, Valvular.

Mitral regurgitation: Mitral Regurgitation is a kind of Valvular heart disease widely known as mitral insufficiency as well. The heart valve (Mitral) does not function properly because of this disorder thus leading to an abnormal leakage of blood to regurgitate into the left atrium of the heart thereby resulting in ascended blood volume and pressure there. The increased blood pressure in the left atrium results in increased blood pressure in the veins stemming from the lungs to the heart (pulmonary veins) and causes the left atrium to expand to provide room for the extra blood leaking back from the ventricle ${ }^{[13]}$.

Doppler echocardiography: Echocardiography aids in the recognition of abnormalities in heart wall motion and in the measure of volume of blood being pumped from the heart with each beat. Besides, abnormalities in the heart's structure, such as defective heart valves, birth defects and enlargement of the heart's walls or chambers, as occurs in people with high blood pressure, heart failure, or impairment of the heart's muscular walls (cardiomyopathy) ${ }^{[13]}$ can as well be diagnosed with the aid of Echocardiography.

A widely used technique for the detection and assessment of the severity of valvular regurgitation is Doppler Echocardiography. Numerous indexes for the 
assessment of severity of regurgitation using color doppler, Pulsed Wave (PW) and Continuous Wave (CW) Doppler have been put forth. The proposed work makes use of Echocardiography image for further investigation and analysis.

Color doppler: Color Doppler is a picture of a blood vessels generated with the aid of standard ultra sound techniques and additionally, a computer transforms the Doppler sounds into colors that are then superimposed on the image of the blood vessel and that characterize the speed and direction of blood flow through the vessel. Detection of regurgitant valve lesions is widely carried out with the aid of Color flow Doppler. The visualization of the origin of the regurgitation jet and its width (vena contracta), the spatial orientation of the regurgitant jet area in the receiving chamber and, in cases of significant regurgitation, flow convergence into the regurgitant orifice is offered by the color flow Doppler technique. The utility, advantages and limitations of Echocardiography and Doppler parameters used in the evaluation of mitral regurgitation severity is shown in the Table 1.

Anisotropic diffusion image segmentation: The process of partitioning an image into several regions where each one is analogous to a homogeneous surface in a scene is known as Image segmentation. Image segmentation is a low-level image processing task, which intends to divide an image into identical regions ${ }^{[16]}$. Many applications employ the color image segmentation. Observation reveals that most existing segmentation algorithms treat color features independently; however it is possible that the result may contain false counters. Due to the reason that the inherent multi-features not only contain non linear relation individually but also comprise inter-feature dependency between R, G and B (or $Y, C_{b}, C_{r}$ ), color image segmentation is more tedious in comparison with grey image segmentation.

In order to accomplish image smoothing and segmentation, numerous models of linear and nonlinear diffusion have been put forth in literature. Numerous researchers have projected nonlinear anisotropic diffusion in their works. A diffusive procedure that is suppressed or stopped at boundaries by selection of locally adaptive diffusion strengths is known as Smoothing. Higher levels of image processing utilize the anisotropic diffusion in the preprocessing stage. The anisotropic diffusion smoothes image interiors to emphasize boundaries for segmentation, eliminates spurious detail to improve the response of edge detection algorithms besides proving efficient at eradicating noise from images. Nevertheless, relaxation processes that implement anisotropic diffusion have a tendency to leave the low frequency objects that are complex to disperse without over-processing the image ${ }^{[17]}$.

Table 1: Echocardiographic and Doppler parameters used in the evaluation of mitral regurgitation severity: Utility, advantages and limitations

\begin{tabular}{lll}
\hline & Utility/advantages & Limitations \\
\hline $\begin{array}{l}\text { Structural parameters: } \\
\text { LA and LV size }\end{array}$ & $\begin{array}{l}\text { Enlargement sensitive for chronic significant MR, important for } \\
\text { outcomes. Normal size virtually excludes significant chronic MR. }\end{array}$ & $\begin{array}{l}\text { Enlargement seen in other conditions. May be } \\
\text { normal in acute significant MR. }\end{array}$ \\
$\begin{array}{l}\text { MV leaflet/support } \\
\text { apparatus }\end{array}$ & $\begin{array}{l}\text { Flail valve and ruptured papillary muscle specific for } \\
\text { significant MR. }\end{array}$ & $\begin{array}{l}\text { Other anormalities do not imply significant MR } \\
\text { Dignos }\end{array}$
\end{tabular}

Doppler parameters:

Jet area-color flow

Simple, quick screen for mild or severe central MR; evaluates spatial orientation of jet.

Vena contracta width Simple, quantitative, good at identifying mild or severe MR

PISA method

Flow quatitation-PW

Quantitative; Presence of flow convergence at Nyquist limit of $50-60 \mathrm{~cm} \mathrm{sec}^{-1}$ alerts to significant MR. Provides both, lesion severity (EROA) and volume overload (R Vol)

Quantitative, valid in multiple jets and eccentric jets. Provides both lesion severity (EROA, RF) and volume overload (R Vol)

Jet profile-CW Simple, readily available

Peak mitral E velocity Simple, readily available, A-wave dominance excludes severe MR

Pulmonary vein flow Simple, Systolic flow reversal is specific for severe MR
Subject to technical, hemodynamic variation; significantly underestimates severity in wallimpinging jets.

Not useful for multiple MR jets; Intermediate values require confirmation. Small values; thus small error leads to large \% error.

Less accurate in eccentric jets; not valid in multiple jets. Provide peak flow and maximal EROA.

Measurement of flow at MV annulus less reliable in calcific MV and/or annulas. Not valid with concomitant significant AR unless pulmonic site is used.

Qualitative; complementary data.

Influenced by LA pressure, LV relaxation, MV area and atrial fibrillation. Complementary data only, does not quantify MR severity. Influenced by LA pressure, atrial fibrillation. Not accurate if MR jet directed into the sampled vein.

CW: Continuous Wave Doppler; EROA: Effective Regurgitant Orifice Area; LA: Left Atrium; PISA: Proximal Isovelocity/Surface Area, LV: Left Ventricle, PW: Pulsed Wave Doppler; MV: Mitral Valve; MR: Mitral Regurgitation; RVol: Regurgitant Volume 


\section{MATERIALS AND METHODS}

One of the chief goals in clinical cardiology is the estimation of severity of mitral regurgitation. Its severity greatly influences clinical decision making ${ }^{[4]}$. Numerous echocardiographic techniques have been put forth for enhanced quantification of valvular incompetence ${ }^{[7]}$. The screening for the existence of mitral regurgitation is commonly carried out with the aid of Color Doppler flow mapping. Prominently, small color flow jets are observed in approximately $40 \%$ of healthy normal volunteers and so are regarded as normal variants. Regurgitant jet area, vena contracta and flow convergence (PISA) are three methods of computing MR severity by color flow Doppler mapping. Even though jet area was the primary method utilized for estimating MR severity it has been found to be less accurate than its latter counterparts and thus the proximal flow convergence method using color Doppler has been recognized as a reliable and accurate quantitative approach.

Jet area: A speedy screening of the presence and direction of the regurgitant jet and a semi-quantitative estimation of its severity can be obtained with the aid of visualization of the regurgitant jet area in the receiving chamber. Various technical, physiologic and anatomic factors have an influence on the size of the regurgitant area and thus modify its accuracy as an index of regurgitation severity ${ }^{[15]}$. Instrument factors, precisely Pulse Repetition Frequency (PRF) and color gain affect the size of a jet. Nyquist limit (aliasing velocity) of 50$60 \mathrm{~cm} \mathrm{sec}^{-1}$ and a color gain that just eliminates random color speckle from non-moving regions is made use of in the standard technique.

Commonly, the large jets that extend deep into the Left Atrium (LA) signify more MR than small thin jets that appear just beyond the mitral leaflets. Owing to numerous technical and hemodynamic limitations noted earlier ${ }^{[15]}$ the correlation among jet area and MR severity is poor. Small eccentric color flow jet area might be detected in patients who have low blood pressure and LA pressure due to chronic severe MR whereas the jet area for hypertensive patients with mild MR is huge. The estimation of severity of MR by "eyeballing" or planimetry of the MR color flow jet area only, is not suggested due to the aforesaid considerations. However, small, non-eccentric jets covering an area $<4.0 \mathrm{~cm} 2$ or $<20 \%$ of LA area trace or mild MR (Table 2) commonly.

Vena contracta: The narrowest part of a jet that appears at or just downstream from the orifice is referred to as the vena contracta. High velocity and laminar flow are characteristics of the vena contracta. It is smaller than the anatomic regurgitant orifice to some extent due to boundary effects. The cross-sectional area of the vena contracta denotes a measure of the Effective Regurgitant Orifice Area (EROA), the narrowest area of actual flow. The size of the vena contracta is not influenced by the flow rate and driving pressure for a fixed orifice.

Table 2: Qualitative and quantitative parameters useful in grading mitral regurgitation severity

\begin{tabular}{|c|c|c|c|}
\hline & Mild & Moderate & Severe \\
\hline \multicolumn{4}{|l|}{ Structural parameters: } \\
\hline LA size & Normal* & Normal or dilated & Usually dilated ${ }^{* *}$ \\
\hline LV size & Normal* & Normal or dilated & Usually dilated** \\
\hline Mitral leaflets or support apparatus & Normal or abnormal & Normal or abnormal & Abnormal/flail leaflet/ruptured papillary muscle \\
\hline \multicolumn{4}{|l|}{ Doppler parameters: } \\
\hline Color flow jet area $\xi$ & $\begin{array}{l}\text { Small, central jet } \\
\text { (usually }<4 \mathrm{~cm}^{2} \text { or } \\
<20 \% \text { of LA area). }\end{array}$ & Variable & $\begin{array}{l}\text { Large central jet (usually }>10 \mathrm{~cm}^{2} \text { or }>40 \% \text { of LA } \\
\text { area) or variable size wall impinging jet swirling in LA. }\end{array}$ \\
\hline Mitral inflow-PW & A wave dominant $\phi$ & Variable & E wave dominant $\phi$ (E usually $1.2 \mathrm{~m} \mathrm{sec}^{-1}$ ) \\
\hline Jet density-CW & Incomplete or faint & Dense & Dense \\
\hline Jet contour-CW & Parabolic & Usually parabolic & Early peaking-triangular \\
\hline Pulmonary vein flow & Systolic dominance $\S$ & Systolic blunting $\S$ & Systolic flow reversal $\uparrow$ \\
\hline \multicolumn{4}{|l|}{ Quantitative parameters $\Psi$ : } \\
\hline VC width $(\mathrm{cm})$ & $<0.3$ & $0.3-0.69$ & $\geq 0.7$ \\
\hline $\mathrm{RVol}\left(\mathrm{mL}\right.$ beat $\left.^{-1}\right)$ & $<30$ & $30-44,45-59$ & $\geq 50$ \\
\hline $\mathrm{RF}(\%)$ & $<30$ & $30-39,40-49$ & $\geq 60$ \\
\hline EROA $\left(\mathrm{cm}^{2}\right)$ & $<0.20$ & $0.20-0.29,0.30-0.39$ & $\geq 0.40$ \\
\hline
\end{tabular}

RF: Regurgitant Fraction; RVol: Regurgitant Volume, VC: Vena Contracta; *: Unless there are other reasons for LA or LV dilation. Normal 2D measurements: $\mathrm{LV}$ minor axis $\leq 2.8 \mathrm{~cm} \mathrm{~m}^{-2}$, LV end-diastolic volume $\leq 82 \mathrm{~mL} \mathrm{~m}^{-2}$, maximal LA antero-posterior diameter $\leq 2 \mathrm{~cm} \mathrm{~m}^{-2}$, maximal LA volume $\leq 36 \mathrm{~mL} \mathrm{~m}^{-2}(2,33,35)$; **: Exception: acute mitral regurgitation; $\xi$ : At a Nyquist limit of $50-60 \mathrm{~cm} \mathrm{sec}^{-1}$; $\uparrow:$ Pulmonary venous systolic flow reversal is specific but not sensitive for severe MR; $\phi$ : Usually above 50 years of age or in conditions of impaired relaxation, in the absence of mitral stenosis or other causes of elevated LA pressure; §: Unless other reasons for systolic blunting (e.g., atrial fibrillation, elevated left atrial pressure); $\Psi$ : Quantitative parameters can help sub-classify the moderate regurgitation group into mild-to-moderate and moderate-tosevere 
Nevertheless, the vena contracta is prone to changes with hemodynamics or during the cardiac cycles if the regurgitant orifice is dynamic. The vena contracta needs to be imaged with high-resolution, zoom views so as to acquire the largest obtainable proximal jet size for measurements. Several studies have shown that the width of the vena contracta is accurate in assessing the severity of MR, either by transthoracic or transesophageal echocardiography.

Proximal isovelocity surface area (Pisa) or flow convergence method: With the assumption that, in the region proximal to a regurgitant orifice, flow is laminar and accelerates smoothly, forming concentric shells of increasing velocity and decreasing surface area, the proximal convergence method is based on the conservation of mass. The PISA method is derived from the above mentioned principle. This region of higher velocity and smaller flow dimension is known as vena contracta ${ }^{[6]}$. Hypothetically, the flow convergence region proximal to a discrete regurgitant orifice in a flat planar surface is a hemispheric volume. The flow in this hemispherical volume accelerates toward the regurgitant orifice along radial streamlines. Concentric hemispheric shells of equal and accelerating velocities (velocity isopleths) constitutes this zone of proximal flow acceleration.

Color flow mapping offers the ability to image one of these hemispheres that corresponds to the Nyquist limit of the instrument. If a Nyquist limit can be chosen at which the flow convergence has a hemispheric shape, flow rate $\left(\mathrm{mL} \mathrm{sec}^{-1}\right)$ through the Regurgitant Orifice (RO) is calculated as the product of the surface area of the hemisphere $\left(2 \pi r^{2}\right)$ and the aliasing velocity $\left(V_{a}\right)$ as:

Flow Rate $(\mathrm{Q})=2 \pi \mathrm{r}^{2} * \mathrm{~V}_{\mathrm{a}}$

Then the regurgitant orifice area ROA is given by:

$\mathrm{ROA}=2 \pi \mathrm{r}^{2}$

where, $r$ represents the radial distance from the orifice to the first alias. The maximal EROA is derived with the assumption that the maximal PISA radius occurs at the time of peak regurgitant flow and peak regurgitant velocity:

$\mathrm{EROA}=\left(6.28 \mathrm{r}^{2} * \mathrm{~V}_{\mathrm{a}}\right) / \mathrm{P}_{\mathrm{k}} \mathrm{V}_{\text {reg }}$

where, $\mathrm{P}_{\mathrm{k}} \mathrm{V}_{\text {reg }}$ is the peak velocity of the regurgitant jet obtained by employing CW Doppler. The product of EROA and the velocity time integral of the regurgitant jet is determined to estimate the regurgitant volume. In this approach, EROA determined is the maximal EROA, which may be slightly larger than EROA calculated by other methods, due to the fact that the PISA calculation proffers an instantaneous peak flow rate. According to the continuity principle, blood flow passing through a given hemisphere needs to pass through the narrowed orifice ultimately ${ }^{[4]}$. Thus, the flow rate through any given hemisphere and the flow rate through the narrowed orifice must be equivalent:

$$
2 \pi \mathrm{r}^{2} \mathrm{~V}_{\mathrm{a}}=\mathrm{A} \times \mathrm{O}
$$

Where:

$\mathrm{A}_{\circ}=$ The area of the narrowed orifice $\left(\mathrm{cm}^{2}\right)$

$\mathrm{V}_{\circ}=$ The peak velocity through the narrowed orifice $\left(\mathrm{cm} \mathrm{sec}^{-1}\right)$

Thus, the area through the narrowed orifice $\left(\mathrm{A}_{\circ}\right)$ can be calculated by rearranging the Eq. 5 :

$$
\mathrm{A}_{\circ}=\frac{2 \pi \mathrm{r}^{2} \mathrm{~V}_{\mathrm{a}}}{\mathrm{V}_{\mathrm{o}}}
$$

As mentioned earlier, the regurgitant volume through an incompetent valve and the flow at the regurgitant orifice are equal. Hence, the regurgitant volume can also be calculated from the ROA and the VTI of the regurgitant signal, with the assumption that the regurgitant orifice does not change throughout the period of regurgitant flow:

$\mathrm{R}_{\mathrm{vol}}=\mathrm{ROA} \times \mathrm{VTI}_{\mathrm{RJ}}$

Where:

$\mathrm{R}_{\mathrm{vol}}=$ Regurgitant volume (cc)

ROA $=$ Effective regurgitant orifice $\left(\mathrm{cm}^{2}\right)$

$\mathrm{VTI}_{\mathrm{RJ}}=$ Velocity time integral of regurgitant jet signal (cm)

The regurgitant volume is deliberated from the simplified method when the mitral regurgitation is eccentric. In this case, the ratio between maximum mitral regurgitant velocity and the VTI of the regurgitant signal is 3.25 . Thus the regurgitant volume is estimated from the regurgitant flow rate and the constant:

$$
\mathrm{R}_{\mathrm{vol}}=\frac{2 \pi \mathrm{r}^{2} \mathrm{~V}_{\mathrm{a}}}{3.25}
$$

Regurgitant Fraction (RF) was expressed as the percent of regurgitant volume $\left(\mathrm{R}_{\text {vol }}\right)$ divided by the total 
stroke volume at the mitral annulus level. The regurgitant fraction would be described as:

$$
\begin{aligned}
\text { FF }= & \frac{\text { FlowThroughTheAorticValve }}{\text { ForwardFlowThroughTheMitralValve }}
\end{aligned}
$$

where, ForwardFlowThroughTheMitralValve $=$ Mitral orifice area $\left(2 \pi r^{2}\right) \times$ Diastolic velocity integral and FlowThroughTheAorticValve $=$ Aortic orifice area $\left(\pi \mathrm{d}^{2} / 4\right) \times$ Systolic velocity integral and the velocity integral of the regurgitant signal is considered to be 3.25 .

\section{RESULTS}

Let us consider a high-quality color Doppler flow images of the flow convergence region with at least one alias were obtained for the calculation of regurgitant flow rate, effective regurgitant flow rate, regurgitant volume and the percentage of backward flow of blood in Mitral regurgitation. In the preprocessing stage, the color Doppler images with RGB color space is converted into $\mathrm{YC}_{b} \mathrm{C}_{\mathrm{r}}$ color space. Followed by, in the second stage, the converted color space image is segmented using non linear anisotropic diffusion method which is used to segment the color spaces exactly. With this the percentage of backward blood flow in mitral regurgitation has been calculated. Figure 1 shows the output representation of anisotropic diffusion segmentation process.

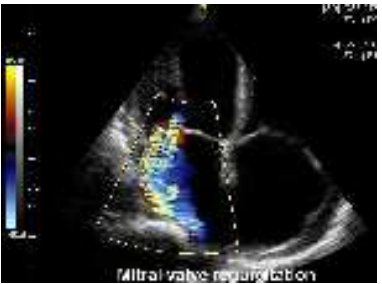

(a)

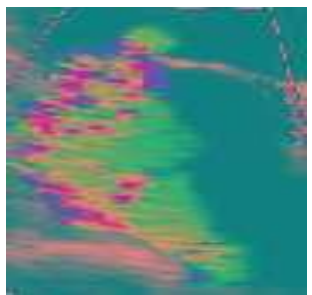

(c)

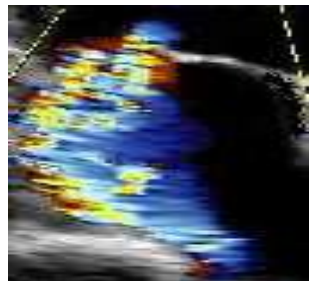

(b)

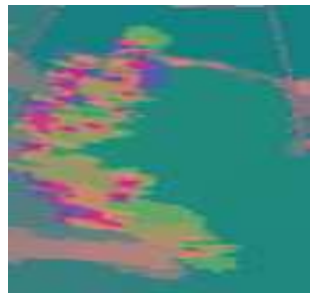

(d)
Fig. 1: Output representation of anisotropic diffusion segmentation process. (a): Mitral regurgitation input image; (b): Region-of-interest; (c): $\mathrm{YC}_{\mathrm{b}} \mathrm{C}_{\mathrm{r}}$ color space converted image; (d): Segmented image
The research shows that the effective ROA calculated based on the analysis of the proximal flow convergence zone, displayed by color Doppler flow mapping image, is feasible and correlates very closely with the true effective regurgitant orifice for a range of different orifice. To apply proximal flow convergence method in the clinical setting, we have calculated effective ROA based on the analysis of the proximal flow convergence zone with mitral regurgitation. The effective measurements of above mentioned parameters for Mild, Severe centric and severe Eccentric mitral regurgitation are detailed in Table 3 and the color Doppler images used for the quantification of mitral regurgitation is shown in Fig. 3.

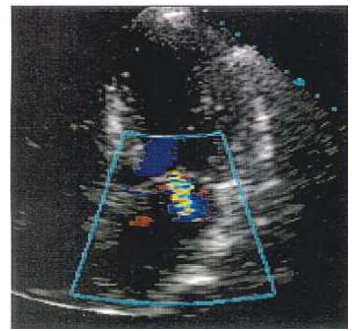

(a)

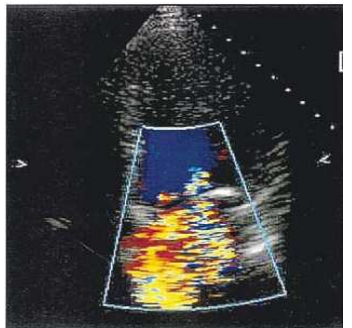

(b)

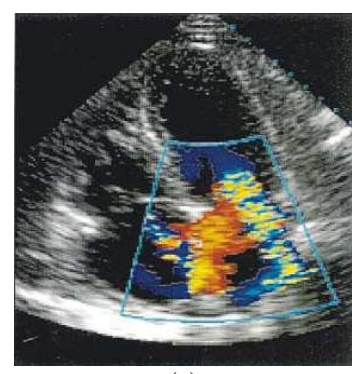

(c)

Fig. 3: Shows the Mitral regurgitation color Doppler images used for Quantification. (a): Mild MR; (b): Severe Centric MR; (c): Severe Eccentric

Table 3: Measured values of the above mentioned parameters of mild, severe centric and severe eccentric mitral regurgitation

\begin{tabular}{lrrr}
\hline & \multicolumn{1}{c}{$\begin{array}{c}\text { Severe } \\
\text { Parameters }\end{array}$} & $\begin{array}{c}\text { Severe } \\
\text { eccentric }\end{array}$ \\
\hline Percentage of blood flow & 3.2200 & 17.1000 & 18.76000 \\
Aliasing velocity $\left(\mathrm{cm} \mathrm{sec}^{-1}\right)$ & 50.0000 & 60.0000 & 60.00000 \\
Peak velocity $\left(\mathrm{cm} \mathrm{sec}^{-1}\right)$ & 72.0000 & 68.0000 & 68.00000 \\
r $(\mathrm{cm})$ & 0.6850 & 1.0110 & 1.24500 \\
Vena contracta width $(\mathrm{cm})$ & 0.2310 & 0.5450 & 1.18500 \\
ROA $\left(\mathrm{cm}^{2}\right)$ & 2.7010 & 6.4100 & 9.73000 \\
Q $\left(\mathrm{cm}^{3} \mathrm{sec}^{-1}\right)$ & 135.0500 & 384.6000 & 583.80000 \\
EROA $\left(\mathrm{cm}^{2}\right)$ & 1.8756 & 5.6550 & 8.85200 \\
A $_{\mathrm{o}}\left(\mathrm{cm}^{2}\right)$ & 1.8756 & 5.6550 & 8.85200 \\
Rvol $\left(\mathrm{mL} \mathrm{sec}^{-1}\right)$ & 41.5500 & 118.3380 & 179.63000 \\
RF $(\%)$ & 0.4550 & 0.49885 & 0.49930 \\
\hline
\end{tabular}




\section{DISCUSSION}

Basically, the hemodynamic estimation of valvular regurgitation is restricted to a semi quantitative grading of invasive or noninvasive parameters which have exposed some correlation with the regurgitant volume. Furthermore, the regurgitant stroke volume, regurgitant flow rate, or regurgitant fraction is determined with the aid of quantitative angiographic technique or the newly developed quantitative color Doppler flow methods.

According to our research, ROA has been established as the elementary parameter in the estimation of valvular incompetence. Nonetheless, lately, the proximal flow convergence method has been put forth as a potential procedure to quantitate regurgitant lesions. Besides supporting the calculation of regurgitant volume and regurgitant flow rate, this technique also aids in obtaining the ROA. With the aid of the principle of conservation of mass, it is possible to determine the instantaneous orifice flow rate on basis of the examination of the converging flow field adjacent to the orifice.

A boundary layer of low velocity flow might result due to viscous forces besides a decrease in the effective surface area of the implicit isovelocity hemisphere. Flow progresses at an angle to the direction of examination on axial images and may cause the underestimation of velocities. Here, we determine the radial distance $r$ with the velocity acquired from the isovelocity hemisphere. This might be responsible for the estimation of flow rate when the first alias was nearer to the orifice. The results of proposed research put forward that the supposition of an isovelocity hemisphere is applicable in the flow convergence zone far away from the orifice where precise estimates of flow rate were achieved. With the aid of these precise measurements we determine the flow rate, effective regurgitant orifice area and the regurgitant volume by utilizing flow convergence method in the mitral regurgitation. An evaluation of the limitations of PISA has been carried out ${ }^{[14]}$. It has been found to be more precise for eccentric jets and for regurgitation having a circular orifice. The effortless identification of the aliasing line of the hemisphere can be done once the image resolution supports the flow convergence to be clearly visible and a Nyquist limit is chosen such that the flow convergence has a hemispheric shape.

To summarize, we have demonstrated that quantitative assessment of the valve regurgitation is achievable with the aid of Doppler flow mapping of the zone of flow convergence proximal to a regurgitant orifice. The utilization of flow convergence region might seem more attractive on theoretical basis rather than the utilization of the features of a turbulent downstream jet.

\section{CONCLUSION}

The precise determination of the severity of the disease is of vital significance with regard to the clinical decision making process for mitral regurgitation. The possibility of surgery necessitates confirmation of severity by a complementary procedure. A novel approach that could precisely and safely quantify mitral regurgitation in theory with the aid of proximal flow convergence method has been proposed in this study. A comparatively greater accuracy was obtained in the quantification mitral regurgitation Doppler image due to the anisotropic diffusion segmentation in the preprocessing stage. We would conclude from our researchers that determination of cardiac output non-invasively by Doppler echocardiography with the aid of the flow convergence method is beneficial. Experimental results have been found to correlate with the several other procedures in existence for cardiac output measurement.

The dynamic nature of lesion and the influence of various hemodynamic and physiologic conditions on it pose a huge challenge for most of the diagnostic techniques working on regurgitation. A comparison of sequential images can now be carried out readily alongside for a more accurate estimation of interval change in the aforesaid adaptive processes and for enhanced timing to carry out the surgery with the aid of the advancements in digital echocardiography. The developments in imaging technologies would facilitate the availability of spatial distribution of the valve regurgitation readily so as to improve measurements of flow convergence, vena contracta and the regurgitant eventually leading to enhancements in the quantization of valvular regurgitation.

\section{REFERENCES}

1. Paige, D.G., 2005. Skin Disease. In: Clinical Medicine, Kumar, P. and M. Clark (Eds.). 6th Edn., Elsevier Saunders Limited, London, UK., ISBN: 0702027634, pp: 1528.

2. Walker, P.G., K. Houlind, C. Djurhuus, W.Y. Kim and E.M. Pedersen, 2000. Motion correction for the quantification of mitral regurgitation using a control volume method. Magnet. Resonance Med., 43: 726-733.

http://www.ncbi.nlm.nih.gov/pubmed/10800038

3. Purcell, E., H. Torrey and R. Pound, 1946. Resonance absorption by nuclear magnetic moments in a solid. Phys. Rev., 69: 37-38. DOI: 10.1103/PhysRev.69.37 
4. Aotsuka, H., K. Tobita, H. Hamada, M. Uchishiba, S. Tateno, K. Matsuo, T. Fujiwara and K. Niwa, 1996. Validation of the proximal isovelocity surface area method for assessing mitral regurgitation in children. Pediat. Cardiol., 17: 351-359. DOI: $10.1007 / \mathrm{s} 002469900079$

5. Enriquez-Sarano, M., W.K. Freeman and C.M. Tribouilloy, 1999. Functional anatomy of mitral regurgitation: Accuracy and outcome implications of transesophageal echocardiography. J. Am. College Cardiol., 34: 1129-1136. http://content.onlinejacc.org/cgi/reprintframed/34/4/1129

6. Schmidt, A., O.C. Almeida Filho, A. Pazin Filho, J.A. Marin-Neto and B.C. Maciel, 2000. Valvular regurgitation by color doppler echocardiography. Arquivos Brasileiros Cardiol., 74. http://publicacoes.cardiol.br/abc/2000/7403/74030 008i.pdf

7. Assef, J.E. et al., 2004. Doppler echocardiographic variables and the type of surgery to be performed in rheumatic mitral valve regurgitation. Arquivos Brasileiros Cardiol., 83. DOI: 10.1590/S0066782X2004001900012

8. Sandeep Bhachu, 2004. A New Method to Quantify Mitral Regurgitation. 4th year Medicine, TSMJ, Volume 5: Review Article, April 2004. http://www.tcd.ie/tsmj/2004/A\%20New\%20Method\%20 to\%20Quantify\%20Mitral\%20Regurgitation.pdf

9. Bisoi, A.K., M.R. Rajesh, S. Talwar, S. Chauhan, R. Ray and P. Venugopal, 2006. Mitral stenosis after duran ring annuloplasty for non-rheumatic mitral regurgitation-A foreign body response? Heart Lung Circulat., 15: 189-190. DOI: 10.1016/j.hlc.2005.08.005

10. Chau, E.M., L.C. Cheng and J.W. Lee, 2001. Severe mitral regurgitation due to mitral valve prolapse associated with Bland-White-Garland syndrome. Hong Kong Med. J., 7: 307-310. http://www.hkmj.org/article_pdfs/hkm0109p307.pdf

11. Liu, K. and A. Priatna, 2001. Flow insensitive black-blood extra cranial MRA by using double-ir and dual-contrast FSE. Proc. Int. Soc. Magnet. Resonance $\quad$ Med., 1919. http://cds.ismrm.org/ismrm-2001/PDF7/1919.pdf
12. Bermejo, J., J.C. Antoranz, M.A. GarcíaFernández, M.M. Moreno and J.L. Delcán, 2000. Flow dynamics of stenotic aortic valves assessed by signal processing of doppler spectrograms. Am. J. Cardiol., 85: 611-617. http://cat.inist.fr/?aModele=afficheN\&cpsidt=1289378

13. Kim, M.S., Y.J. Kim, H.K. Kim, D.A. Kwon, D.H. Kim, J.B. Seo, H.M. Yang, D.H. Choi and D.W. Sohn, 2008. Systolic mitral annular velocity as a simple marker of left ventricular peak systolic $\mathrm{dP} / \mathrm{dt}$ in patients with significant mitral regurgitation. J. Cardiovasc. Ultrasound, 16: 48-53. http://www.koreamed.org/SearchBasic.php?DT=1 $\& \mathrm{RID}=435369$

14. Simpson, I.A., T. Shiota, M. Gharib and D.J. Sahn, 1996. Current status of flow convergence for clinical applications: is it a leaning tower of PISA. J. Am. College Cardiol., 27: 504-509. DOI: 10.1016/0735-1097(95)00486-6

15. Sahn, D.J., 1988. Instrumentation and physical factors related to visualization of stenotic and regurgitant jets by Doppler color flow mapping. J. Am. College Cardiol., 12: 1354-1365. http://content.onlinejacc.org/cgi/content/abstract/12 /5/1354

16. Duda, R. and P. Hart, 1973. Pattern Classification and Scene Analysis. Bayes Decision Theory. John Wiley and Sons, USA., ISBN: 13: 9780471223610 , pp: 482.

17. Seramani, S., Z. Jiayin, C.K. Luk, N. Malmurugan and A. Nagappan, 2008. Denoising of MR images using non linear anisotropic diffusion filtering as a preprocessing step. Int. J. BioSci. Technol., 1: 17-21. http://www.ijbst.org-a.googlepages.com/IJBST_1_ 1_17-21.pdf 